Published in final edited form as:

J Am Acad Child Adolesc Psychiatry. 2012 October ; 51(10): 1052-1065. doi:10.1016/j.jaac.

2012.08.003.

\title{
Effects of a Brief Early Start Denver Model (ESDM)-Based Parent Intervention on Toddlers at Risk for Autism Spectrum Disorders: A Randomized Controlled Trial
}

\author{
Dr. Sally J. Rogers, Ph.D., \\ University of California-Davis (UC Davis) Medical Investigation of Neurodevelopmental Disorders \\ (MIND) Institute
}

Dr. Annette Estes, Ph.D., University of Washington

Dr. Catherine Lord, Ph.D., Weill Cornell Medical College

Dr. Laurie Vismara, Ph.D., University of California-Davis (UC Davis) Medical Investigation of Neurodevelopmental Disorders (MIND) Institute

Dr. Jamie Winter, Ph.D., University of Washington

Dr. Annette Fitzpatrick, Ph.D., University of Washington

Dr. Mengye Guo, Ph.D., and

University of Washington

Dr. Geraldine Dawson, Ph.D.

Autism Speaks and the University of North Carolina

\begin{abstract}
Objective-This study was carried out to examine the efficacy of a 12-week, low intensity (onehour-per-week of therapist contact), parent-delivered intervention for toddlers at risk for autism spectrum disorders (ASD) ages 14-24 months and their families.
\end{abstract}

Method-A randomized controlled trial involving 98 children and families was carried out in three different sites investigating the efficacy of a parent delivery of the Early Start Denver Model (P-ESDM) (which fosters parental use of a child-centered responsive interaction style that embeds many teaching opportunities into play) compared to community treatment as usual. Assessments

\footnotetext{
(C) 2012 American Academy of Child \& Adolescent Psychiatry. Published by Elsevier Inc. All rights reserved. Correspondence to Sally J. Rogers, Ph.D., UC Davis MIND Institute, Psychiatry and Behavioral Sciences, 2825 50th Street, Sacramento, CA 95817; sally.rogers@ucdmc.ucdavis.edu.

Disclosure: Drs. Rogers, Vismara, and Dawson have received royalties from the sale of books and other printed Early Start Denver Model (ESDM) materials. Drs. Estes, Lord, Winter, Fitzpatrick, and Guo report no biomedical financial interests or potential conflicts of interest.

Publisher's Disclaimer: This is a PDF file of an unedited manuscript that has been accepted for publication. As a service to our customers we are providing this early version of the manuscript. The manuscript will undergo copyediting, typesetting, and review of the resulting proof before it is published in its final citable form. Please note that during the production process errors may be discovered which could affect the content, and all legal disclaimers that apply to the journal pertain.
} 
were completed at baseline and 12 weeks later, immediately after the end of parent coaching sessions.

Results-There was no effect of group assignment on parent-child interaction characteristics or on any child outcomes. Both groups of parents improved interaction skills and both groups of children demonstrated progress. Parents receiving P-ESDM demonstrated significantly stronger working alliances with their therapists than did the community group. Children in the community group received significantly more intervention hours than those in the P-ESDM group. For the group as a whole, both younger child age at the start of intervention, and a greater number of intervention hours, were positively related to degree of improvement in children's behavior for most variables.

Conclusions-Parent-implemented intervention studies for early ASD have thus far not demonstrated the large effects seen in intensive treatment studies. Evidence that both younger age and more intervention hours positively affect developmental rates has implications for clinical practice, service delivery, and public policy.

\section{Keywords}

Early Start Denver Model (ESDM); early intervention; toddler; parent-child interaction; autism

\section{Introduction}

Efficacy of very early intervention for improving short-term outcomes in autism spectrum disorders (ASD) has led scientists to develop diagnostic measures for children under the age of 2 in order to begin treatments as early as possible. ${ }^{1}$ Practice, theory (attachment), and research have all emphasized the importance and efficacy of parent-delivered interventions for children with developmental difficulties early in life. ${ }^{2}$ Studies of parent-child interactions in ASD have found that, on average, parents of children with autism are as responsive to their children as parents of children with other or no diagnoses. ${ }^{3-4}$ Additionally, parental sensitivity and responsivity to children's cues have been found to predict language outcomes in ASD, as they do in typical development. ${ }^{5-7}$ Thus, the effects of parent-delivered toddler interventions are likely mediated by the quality and quantity of parent-child interactions.

The inclusion of parents (as well as other family members) has been identified as an essential component of effective early intervention programs, ${ }^{8}$ and many autism studies using single subject designs have demonstrated the positive effects of parent-delivered interventions for reducing problem behaviors, improving children's nonverbal and verbal communication skills, and increasing appropriate play and imitation skills. ${ }^{9-18}$ Parentimplemented intervention leads to better generalization and maintenance of skills than therapist-implemented intervention. ${ }^{19}$

However, few studies have examined the impact of parent-delivered intervention on outcomes for children with ASD younger than three years of age. One empirically supported intervention approach built for children with ASD younger than 3 is the Early Start Denver Model (ESDM), a developmental, relationship-based intervention that fuses approaches validated by the science of child development and the science of applied behavior analysis. ${ }^{20-21}$ A randomized controlled trial of the ESDM of children ages 18-30 months, delivered by trained interventionists in child homes for 15-20 hours-per-week for two years, demonstrated significant group effects on IQ, language, and adaptive behavior. ${ }^{20}$ The extent to which parents can deliver ESDM was tested by Vismara et al. using a single subject design with eight children with ASD under the age of $3 .^{22}$ The study documented positive 
gains in parent learning of the techniques, and marked gains in child social attention, imitation, and spontaneous language.

Recently, several randomized controlled studies of parent-delivered interventions for young children with ASD have been reported. Several of these report parent behavior change but lack of overall group effects on children's developmental scores and autism symptoms 23-, 25 . Kasari ${ }^{23}$ reported some behavioral gains but no data regarding changes on standardized measures of development or primary autism symptoms.

\section{Purpose of the Present Study}

The present study was designed to examine the effect of P-ESDM with a rigorous design, using the same curriculum, procedures, and manual as in the earlier P-ESDM study, with a much larger and younger multisite sample, a randomized controlled group design, and a more elaborate measurement system. ${ }^{22}$ The primary goal of the study was to determine whether P-ESDM was more effective for improving outcomes of children with ASD than community treatment as usual.

\section{Hypotheses}

1. Children receiving 12 weeks of the P-ESDM will demonstrate greater gains in social communication and developmental quotients than will those in the community intervention group.

2. Parents receiving 12 weeks of P-ESDM parent training will show more skilled use of interactive techniques measured by the P-ESDM Fidelity measure than will parents in the community intervention group.

3. Two child pre-intervention variables that will moderate the effects of P-ESDM on child social and developmental outcomes are social orienting and imitation, key behaviors that support social learning in typically developing pre-verbal toddlers and two of the earliest distinguishing features of ASD in toddlers ${ }^{24-25}$

4. Parents receiving P-ESDM will demonstrate more positive working relations with their primary therapist than will parents in the community intervention group.

\section{Method}

\section{Participants}

Recruitment-We recruited toddlers at risk for ASD, ages 12-24 months, and their families living within 30 minutes of the three sites (Sacramento, Seattle, and Ann Arbor), primarily from pediatricians, regional centers for developmental disabilities, and university autism clinics and research programs. Inclusion criteria included: met risk criteria for ASD on two screeners and met criteria for ASD in a clinical assessment, was ambulatory (crawling or walking), had developmental quotients (DQ) of 35 or higher, and had English as one language spoken daily in the home. Exclusion criteria included parental self-reported significant mental illness or substance abuse, child significant medical conditions including cerebral palsy, gestational age of less than 35 weeks, genetic disorders related to developmental disabilities, DQ below 35, and current or previous enrollment in intensive 1:1 autism intervention of more than 10 hours per week.

Enrollment-The enrollment process began with a telephone screen using two autism screening measures administered by phone to parents of 228 referrals who met initial inclusion/exclusion criteria. Of these 228, 57 were discontinued because they did not meet screening criteria or by choice. One child was excluded because of DQ below 35 during 
initial screening. One hundred sixty six children met autism risk criteria on both screeners and were evaluated diagnostically. Of these, 118 children met inclusion criteria for developmental quotient and for ASD symptoms (the cutoff score on the Autism Diagnostic Observation Scale for Toddlers [ADOS-T] and the clinical judgment of ASD of two independent clinicians based on observation and scores). Furthermore, level of ASD symptoms was quite stable over the 3 month period, with all but one child continuing to meet ADOS-T cutoff scores and clinical judgment for ASD at the 12 week follow-up. Twenty families declined to enroll, and 98 families enrolled in the study and were randomized to P-ESDM treatment or community treatment groups. Following the enrollment visit (Time 1, or T1), children and parents returned for a second evaluation session to gather the remaining measures. Parents were informed of the randomization assignment at this visit, and received diagnostic feedback, a clinical diagnostic report, and detailed referral information about community-based services. Twelve weeks then passed during which families received intervention, followed by a re-assessment (Time 2, or T2), again involving two assessment visits, feedback, reports and recommendations to the families.

Family incomes, ethnicity, and educational levels of the families are included in Table 1. Twenty four of the 98 children had a sibling with ASD, 9 in the ESDM group and 15 in the community group. In addition, 26 children had siblings with another developmental disorder, 10 in the ESDM group and 13 in the community group: including speech or other developmental delays, learning disabilities, seizure disorders, cerebral palsy, Down syndrome, or intellectual disability.

Randomization-Immediately after families consented to enroll, staff members contacted the project's Data Coordinating Center (DCC) at University of Washington for an ID number and group assignment. Children were randomized using a computer algorithm based on pre-specified blocks based on age (12-20 months or greater than 20 months), gender, and DQ (less than 60,60 or greater). Blocks were monitored and a difference of 3 between intervention/control resulted in a compensatory assignment in order to maximize equal sample in both groups.

Measures

Screeners-Early Screening of Autistic Traits Questionnaire (ESAT) is an autism risk parent interview that has been tested in several studies, including a population-based study of 31,000 14-15 month old children in the Netherlands. This tool was used for children 1215 months. ${ }^{26}$

Infant Toddler Checklist (ITC) is a broadband parent questionnaire developed to determine risk for communication disorders including ASD. The ITC is a standardized tool that has both screening cutoffs and standard scores at monthly intervals from 6 to 24 months based on a normative sample of over 2,188 children. ${ }^{27}$ This screener was used with all children in the study.

The Modified Checklist for Autism in Toddlers (M-CHAT) is a 23-item parent questionnaire developed to screen for autism symptoms in children ages 16-30 months. ${ }^{28}$ The M-CHAT has been validated in two large validation studies. ${ }^{29-30}$ This tool was used for children 16-24 months.

ASD Diagnosis-Autism Diagnostic Observation Scale for Toddlers (ADOS-T) was used at both assessment points for all children with permission. ${ }^{31}$ The tool uses a semistructured observational assessment that provides a number of opportunities for interaction 
(e.g., play, turn-taking games, looking at books, etc.) and measures social and communicative behaviors, as well as repetitive behaviors diagnostic of autism on a scale of 0-3. The full range of scores was used, including 3's, to maximize the range of each item. Subtotals were computed separately for the two domains, Social Affect and Restricted, Repetitive Behaviors. ${ }^{32}$ Lab personnel were trained to $85 \%$ reliability on the full range of scores. Inter-observer reliability at individual sites was assessed on at least $20 \%$ of interviews, and any deviation from standard led to retraining.

Developmental Measures-Mullen Scales of Early Learning (MSEL) is a standardized, normed developmental assessment for children aged birth through 68 months. ${ }^{33}$ It provides an overall index of ability, the Early Learning Composite and subscale scores of Receptive Language, Expressive Language, Visual Reception, and Fine Motor skill. The MSEL was administered at enrollment and 12 weeks later, at the end of intervention, as a dependent measure of treatment efficacy. The MSEL was administered strictly according to standard procedures across the three sites, and administration practices were monitored throughout. Reinforcers for child attention and cooperation were used as needed to support children's motivation and cooperation. Twenty percent of the assessments were scored by two raters for reliability checks. Because the standard t scores on the MSEL have a floor of 20, and many subjects had scores at the floor on individual subscales, we created three developmental quotient scores (DQ; DA/CAx100) from the subscale developmental age (DA) equivalents to use as measures of intervention efficacy: a verbal developmental quotient (Verbal DQ) comprised of the two language scales; a nonverbal developmental quotient comprised of the Visual Reception and Fine Motor scales (Nonverbal DQ); and an overall developmental quotient created from these four subscales.

\section{MacArthur-Bates Communicative Development Inventory: Words and gestures} (MCDI) is a 396-word parent report vocabulary checklist used to capture the expressive words, gestures, and receptive vocabulary a child has demonstrated in the past week. ${ }^{34}$ This is a psychometrically strong instrument, and raw scores in young children with ASD are concurrently associated with communicative word use in language samples $(r=.67$ to . 72). ${ }^{35}$ This was gathered at both time points and raw scores were used as a dependent measure of treatment efficacy.

Vineland Adaptive Behavior Scales, Second Edition (VABS II) examines four domains of adaptive behavior: communication, self-care, social and motor skills. ${ }^{36}$ The VABS-II has excellent psychometric properties. It was administered to the primary caretaker by telephone at both assessment points. The dependent measures of treatment efficacy were the age equivalent scores and the developmental quotients for the four domains.

\section{Child Moderating Variables}

Imitation: Twelve imitation tasks were administered in a playful 10 minute or less probe that examined familiar and novel object imitations and manual and oral-facial gestural imitations. ${ }^{37}$ This scale has been used in several previous autism studies. These were live coded using one of three scores: full pass (2), partial pass (1), and failure (0), resulting in a total imitation score.

Orienting to social, nonsocial and joint attention stimuli: A brief task developed by Dawson (2004) assessed child social engagement. ${ }^{25}$ The task involved child orienting to three types of probes: four "social" noises (e.g., human-produced; voice, finger snap, clap, and hum) and (b) four nonsocial noises (timer ticking, phone beep, sandpaper scratch, light switch click), and (c) four joint attention bids. All sounds were delivered the same decibel level by a lab assistant. The observing experimenter recorded each child's head turns or gaze 
turns towards the sound within 10 seconds. The four joint attention bids were administered by the experimenter, seated in front of the child. Two bids involved name, head turn, and point, and the other two involved name and head turn without pointing. Scores ranged from 0-4 for each of the 3 tasks.

\section{Parent Measures}

General Family Demographic Data: A general demographic questionnaire was administered. It contains questions concerning family composition, family size, number of people in the house, parents' educational levels, parents' occupations, and annual gross incomes.

The Symptom Checklist-90-R (SCL-90-R) is a widely used 90-item, 15-minute questionnaire designed for both clinical and research use. It was used as a psychiatric screener in this study to provide information about self-reported mental health difficulties in the parents. ${ }^{38}$ Each item is scored on a 5-point scale; total scores were the variable of interest.

ESDM Parent Fidelity Tool: (Rogers, Dawson, Vismara, unpublished material, 2012) The P-ESDM Fidelity tool is a Likert- based 5 point rating system of 13 parent behaviors that define the child-centered, responsive interactive style used in ESDM. ${ }^{20}$ These behaviors are: management of child attention, quality of behavioral teaching (use of clear antecedentbehavior-consequence events and efficient teaching strategies embedded in the play), adult ability to modulate child affect and arousal, management of unwanted behaviors using positive approaches, use of turntaking/quality of dyadic engagement, giving child choices, optimizing child motivation for participation in activity, parent display of positive affect, parent sensitivity and responsivity to child communications, parent use of multiple and varied communicative functions, appropriateness of parent language for child's language level, parent use of flexible joint activity routines with theme and variation in activities, smooth transitions between activities that maximize child interest and engagement. Parentchild play interactions with a specified set of toys and the instruction to: "play as you typically do at home" were video-recorded at both assessment points. Expert ESDM therapists blind to group assignment coded parent behaviors by observing videos of 10 minutes of these parent-child play interactions. Total score was the variable used for analysis and could range from 14 to 60 , with a higher score reflecting interactions closer to ESDM principles.

Working Alliance Scale for Interventions with Children. ${ }^{39}$ : This measure was used to examine the responses of families to the primary early intervention therapist working with them at the end of the 12 weeks. It was created specifically for early intervention by adapting an existing working alliance scale, and the authors reported strong internal consistency among scale items (alpha $=0.91)$ and variability in the range of reported scores.

Child Intervention Hours: Amount of intervention of all types was examined twice: at T1, which included all hours received up to that point, and T2, which involved the average number of hours received per week between T1 and T2. Data were gathered via a telephone interview with each family using an adaptation of the CPEA Intervention History interview (unpublished). At each point, parents were asked for every type of intervention the child was receiving, the date the treatment began and ended, the number of hours weekly the child received it, and the number of adults and children present during each of these interventions. From this data, we calculated the intensity of each intervention: the number of weeks in length between the date began and date ended, minus the length of breaks in weeks, multiplied by the number of hours per week the child received it, and then multiplied by the 
ratio of adults to children in the intervention. (For example, 8 weeks of 1:1 treatment 4 hours per day, 5 days per week $=160$ hours. Eight weeks of group treatment 4 hours per day, 5 days per week, with an adult:child ratio of 1:3 $=53.33$ hours.) Intervention types included: applied behavior analysis (ABA), occupational therapy (OT), physical therapy (PT), Speech/ language, Treatment and Education of Autistic and related Communication Handicapped Children (TEACCH), Developmental, Individual Differences, Relationship-based approach (DIR), play therapy, home session with infant-toddler educators, and infant-toddler treatment groups. We included the P-ESDM hours for children in the experimental group. We did not include nutritional interventions, day care, or community recreation classes. At $\mathrm{T} 1$, there was no significant difference in the amount of cumulative treatment hours received by children in the two groups (community group mean=11.06 ( $\mathrm{SD}=3.13$ ), P-ESDM 16.16 (4.97), $\mathrm{p}=.38$ ). Twelve weeks later, at $\mathrm{T} 2$, there was a significant difference in the number of treatment hours children were receiving weekly (P-ESDM 1.48 (1.96); community group 3.68 (3.91), $p<.05$, as shown in Table 1.

\section{Treatment Condition}

The P-ESDM consisted of 12 consecutive sessions, each session lasting one hour. Intervention sessions were conducted in the three university clinics by highly experienced and credentialed therapists trained to fidelity by the authors who monitored it quarterly throughout the study. A detailed parent training curriculum, a specific coaching intervention method, and a therapist fidelity measure defined the approach. ${ }^{40-41}$ Therapist fidelity average score with the child was a mean of 4.47 (s.d. =.24) measured on the 14-item fidelity tool with scores ranging from $1-5$. Therapist fidelity average score in coaching interactions with the parent was a mean of 3.62 (s.d. =.25), measured on 13 items with scores ranging from 1-4.

Session 1 was devoted to developing children's learning objectives and Sessions 11 and 12 to maintenance after treatment and review of progress. In sessions 2-10, parents were coached in each of 10 intervention techniques from the ESDM, addressing one new parent skill each week and refining those taught earlier. The 10 topics were: a) increasing child's attention and motivation; (b) using sensory social routines; (c) promoting dyadic engagement and joint activity routines; (d) enhancing nonverbal communication; (e) building imitation skills; (f) facilitating joint attention; (g) promoting speech development; (h) using antecedent-behavior-consequence relationships (“ABC's of learning”); (i) employing prompting, shaping, and fading techniques; and (j) conducting functional assessment of behavior to develop new interventions.

Sessions began with a 5-minute warm-up period of parent-child interaction within the context of a parent or child-chosen play activity. Then therapists introduced the new topic each week through verbal description and written materials from the manual and briefly modeled the interaction skill with the child in play. Following this demonstration, the therapist and parent briefly discussed what the parent observed, and the parent practiced the technique in play with the child while the therapist provided coaching, encouragement, and feedback on technique use. Then the parent was coached in the skill in several different activities (e.g., books, feeding, dressing or changing, toy play, and social play) until the parent demonstrated the technique at an implementation rate of $80 \%$ or higher. Each session concluded with the therapist and parent discussing use of the new skill in various activities and settings at home and in the community. The parent left with self-instructional manual materials on the target technique to review. ${ }^{40}$ Each session was videotaped for clinical supervision and fidelity coding of parent and therapist. 


\section{Results}

\section{Analytic approach}

Descriptive statistics of baseline characteristics of study participants included frequency (\%) and means and standard deviations (SD) for categorical and continuous variables, respectively. For group comparisons on child outcome variables, we used linear regression models to (1) predict the T2 outcome scores adjusting for the baseline score, the group assignment and possible confounders and to (2) test the main effect of group assignment. We ran the model in two ways: first adjusting only for the time 1 variables in the model for the time 2 variable, and second involving adjustments for those variables in which there were initial significant differences between groups. We also examined the effect of baseline age, imitation, social orient, nonsocial orient and joint attention in predicting the ADOS and Mullen scores in the adjusted models. Pre-specified moderators, imitation and social orienting, were evaluated by testing the interaction between these variables and group assignment in the adjusted model. Given that the logic model of the project assumes that parent behavior is the causal mechanism for changes in child behavior, group effects on measures of parent behavior were examined at both time points, and relations between parent behavior and child symptoms and development examined. The parent working alliance score was only measured at visit 2 and group differences were evaluated.

\section{Descriptive data}

Most demographic child and family variables, presented in Table 1, were distributed equally for P-ESDM and community intervention groups at baseline. Child variables gathered at the two assessments, $\mathrm{T} 1$ and $\mathrm{T} 2$, are presented in Table 2. While the two groups were well matched on most variables, there was a significant difference in the Time 1 modified ADOS Social Affect score $(p<.02)$. Children in the community intervention group had a higher mean ADOS Social Affect score (mean 34.12, SD 8.69), indicating more severe social affect symptoms than did children randomized into P-ESDM (29.45, SD 9.16). The groups also differed significantly in their initial imitation scores and nonsocial orienting scores. In addition, at $\mathrm{T} 2$, but not $\mathrm{T} 1$, we found significant differences in number of intervention hours received between groups.

\section{Effects of group assignment on child behavior}

There was improvement in both groups over the 12 weeks (controlling for change expected with age by using DQs) as reflected in approximately a 10-point increase in verbal DQ, a 45 point increase in overall DQ, and decreasing ADOS Social Affect scores.

Examination of the model adjusting only the $\mathrm{T} 1$ score demonstrated no significant differences related to group assignment on any of the variables listed in Table 2. After adjusting the model for T1 differences in Modified ADOS Social Affect, Imitation and Nonsocial Orienting scores, there continued to be no significant differences related to group assignment on any variable except the T2 Modified Social Affect score, for which the community group showed greater improvement than the P-ESDM group (estimated difference between P-ESDM and community group=3.43, $\mathrm{SD}=1.72, p=.05$ ).

As a pre-specified analysis, the effect of baseline age was also assessed in the linear model and it was found to be a significant predictor of T2 Mullen DQ, independent of group assignment and other baseline variables we adjusted for. The estimated effect for baseline age in predicting the T2 Mullen DQ is -1.20 ( $p$ value $=0.002$ ), indicating toddlers with younger age had a greater increase in the Mullen DQ, after adjusting for baseline Mullen DQ, assignment, baseline Modified ADOS Social Affect, Nonsocial Orient, and Imitation scores. 


\section{Effect of intervention hours on change in child outcomes}

We examined the treatment hours reported by the parents at the start of intervention and 12 weeks later. (All outliers were removed from this before analysis; the range was 0 to 15.9 hrs). There was no relationship between severity of ADOS scores and severity of Mullen DQ on number of hours of treatment at either time point (all $\mathrm{r}^{2}$ less than absolute value of 0.20 ). While the amount of treatment was not expected to differ between the groups, in fact, the community group was receiving significantly more hours of intervention at the end of the 12-week period than was the P-ESDM group. A set of linear models was built to predict the change in the outcome scores using the hours of independent intervention per week at Time 2, with T1 baseline hours of intervention, age and modified ADOS Social Affect as independent covariates (Table 3). We used the $\mathrm{T} 2$ number of intervention hours because the regency of the $\mathrm{T} 2$ hours was considered to have the most potential for affecting short-term child change. The estimated coefficient of the group assignment for the variables listed in Table 2 changed but remained non-significant after adding the intervention hours (results not shown here). After adjusting for intervention hours, the group difference reported above involving change in ADOS Social Affect score was no longer significant.

We then conducted a new analysis in which we combined groups to examine effect of intervention hours on child outcomes adjusted for baseline age, ADOS and baseline value of the specific score. As shown in Table 3, intervention hours had a significant main effect on the change scores for ADOS Restrictive and Repetitive, Mullen Overall DQ, Mullen Verbal DQ, MCDI Vocabulary Comprehension, and Nonsocial Orienting in the pooled sample. When stratified by assignment group, intervention hours were not associated with the child outcomes in either group, except for MCDI vocabulary comprehension and production in the P-ESDM group. This is probably due to the small sample size in each group. There were no significant interactions between intervention hours and group assignment for all the child outcomes, except for MCDI vocabulary production. Although receiving more intervention hours showed some improvement in child outcomes, such as Mullen overall DQ and Verbal DQ, its effect on modified ADOS social affect was ambiguous, with a worsen effect in PESDM group (estimated effect $=0.65, p>0.05$ ) and an improvement effect in community group (estimated effect $=-0.56, p>0.05$ ). Since our trial was not designed to evaluate its effect on child outcomes, we should interpret these results with caution.

\section{Moderators of child change}

Two potential moderators of child change, Imitation and Social Orienting, were hypothesized a priori to influence the association between group assignment and T2 Mullen and ADOS scores. We found no evidence of moderating effects of these two variables on child responses to the P-ESDM treatment. However, Imitation and Nonsocial Orienting had main effects on outcomes over time for the entire group of 98 children. As shown in Table 4, Nonsocial Orienting was a significant predictor of both changes in Mullen DQs and on decreases in Modified ADOS Social Affect score. Social Orienting was a significant predictor of reduction of ADOS Restrictive and Repetitive Behavior score. However, this is likely due in part to the correlation among these variables: $\mathrm{r}=-.30, p=.003$ between Nonsocial Orienting and T1 ADOS Social Affect, $\mathrm{r}=.35, p<.001$ between Nonsocial Orienting and T1 Mullen DQ, and r=-.17, $p=.09$ between Social Orienting and T1 ADOS Restrictive and Repetitive Behavior scores.

\section{Parent acquisition of child-centered interaction and communication skills}

Group differences in fidelity scores-We next examined group differences in change from T1 to T2 in the parents' use of the parent-child interaction techniques measured by the P-ESDM Fidelity scores using linear regression. As seen in Table 5, the groups of parents were equivalent at $\mathrm{T} 1$, and both groups of parents demonstrated significant gains in use of 
these interaction skills over the 12-week period. The follow-up score in the P-ESDM group was larger than that of the community group at $\mathrm{T} 2$ but was not significant after adjusting for baseline age and ADOS Social Affect score $(p=.19)$. The pre-post differences in the PESDM group showed a large effect size (.57) compared to the community group's moderate effect size (.37). Parents' T2 scores showed little relationship to their T1 scores, for either group of parents ( $\mathrm{r}=.28$ for each group).

\section{Relationship between P-ESDM Fidelity scores, child performance, and child} change-Our final analysis of the P-ESDM Fidelity scores examined relations between Fidelity scores and child performance for the entire group of children. At T1, P-ESDM Fidelity scores $(\mathrm{n}=81)$ demonstrated moderate negative correlations with ADOS scores (Social Affect $r=-.36$, ADOS RRBs $r=-.35$ ) and moderate positive correlations with MSEL scores (verbal $\mathrm{DQ}=.36$, nonverbal $\mathrm{DQ}=.34$, full scale $\mathrm{DQ}=.40$ ), though this same relation was not observed at Time 2 . There were no significant relationships between parent change scores on the Fidelity measure during the 12 weeks and child change scores during that same period of time.

\section{Effect of group assignment on Parent Working Alliance}

Parental working alliance with the primary early intervention therapist was measured at the end of the 12-week intervention period. Due to the presence of outliers, we used robust regression (bi-square to predict working alliance adjusting for other covariates. The PESDM group parents (mean=5.60, $\mathrm{SD}=.82$ ) reported a significantly stronger working alliance with their primary therapist than did the community intervention group (mean=5.23, $\mathrm{SD}=1.1) p$ value .06 .

\section{Discussion}

We predicted that 12 weeks of parent-implemented P-ESDM would result in (1) greater parent acquisition of ESDM intervention skills, and (2) greater developmental gains and reduced core autism symptoms in toddlers with ASD, compared to treatment as usual. Contrary to our first hypothesis, families in P-ESDM did not demonstrate significantly higher P-ESDM Fidelity scores than did the community treatment group. Contrary to our second hypothesis, we did not find group differences on any of the primary child outcome variables after the 12-week intervention. Our third hypothesis predicted that social orienting and imitation, fundamental skills for child social and communication learning, would moderate the child effects of P-ESDM. This hypothesis was not supported. The two orienting variables involving response to social and nonsocial stimuli predicted change in autism symptoms and Mullen developmental quotients in both groups of children, likely due in part to shared variance. Our fourth hypothesis, that parents receiving ESDM coaching would demonstrate stronger working alliances with their primary therapist than would parents receiving community intervention, was supported.

Additional analyses yielded several important findings about the measures, design, and effects of the study. First, higher parent P-ESDM Fidelity scores at the start of the study were significantly related to children's milder ASD symptoms and higher developmental scores.. This relationship between P-ESDM Fidelity scores (reflecting parent sensitivity and responsivity to children's communications and interests and their ability to create effective learning experiences) and their children's development and autism symptoms was expected, based on findings reported from previous studies; it formed the logic for the treatment model used in this study. 6,42

In terms of child change across the 12 weeks, we found that both groups made significant gains in the rates of acquisition of developmental skills and reduction in core autism 
symptoms, even though $95 \%$ of children continued to meet ADOS-T criteria for ASD. Measures of both ASD symptoms and of developmental impairment are continuous measures, and children showed improvement through lessened severity of scores even though they continued to meet diagnostic criteria.

In addition, we found that the degree of child gain was related to (1) the number of hours of intervention received and (2) younger child age. Number of hours of intervention powerfully predicted amount of change on all dependent variables, including lessening of autism symptoms. Age at initiation of intervention also had important effects on 12-week outcomes, with those toddlers closer to 24 months at enrollment showing smaller amounts of change in developmental rates (but not autism symptom reduction) than did those who were younger. These two findings, if replicated, have important implications for early intervention policy and practice for toddlers at high risk for ASD. A point we return to below.

Given that the parents in the P-ESDM group were being actively coached in the P-ESDM techniques, and made significant gains across the study, why were there no differences after 12 weeks between the ESDM and the community treatment parent groups? Several confounds in the study design may be responsible. A primary confound involved the amount of intervention that the community group received and the effects of intervention hours on child progress. While $\mathrm{T} 1$ amounts of intervention did not differ between groups, the community group was receiving significantly more intervention than the P-ESDM group 12 weeks later (almost double). This may have been influenced by the RCT design itself, in two ways. Our efforts at earliest diagnosis, provision of clinical reports, referral information, and parent information about ASD may have facilitated the community group's rapid enrollment in other interventions. Second, it is possible that the randomization assignment to the comparison group further motivated these already motivated families to secure other services for their child as quickly as possible. (P. Yoder, personal communication, with permission).

A second and related confound is that it is quite likely that the services the community treatment group received involved parent coaching, since this is the most common way for Birth to Three Services to be delivered, given the Part $C$ federal requirements. The content they received may also have been similar to P-ESDM. Information in following children's leads and responding to their communicative efforts is a widespread technique for helping young children with delays develop speech and language. Play-based interventions are a very typical delivery format for Part $\mathrm{C}$ home-based services. Thus, it is quite possible that parents in the community group were receiving input similar in kind to what the P-ESDM parents were receiving, at a higher intensity, which could explain their increases in the ESDM parent fidelity scores and their children's progress.

A third design weakness involved the measurement schedule. The P-ESDM group parents were learning the skills throughout the 12-week period that also defined the pre-post child measurement period, and parents mastered the techniques at around weeks $8-9$. ${ }^{22}$ Thus, the children in the P-ESDM group were not receiving the "full dosage" of the intervention until the very end of the measurement period. There may not have been enough time for the parent skill changes to affect child behavior measurably. Taking an additional set of measurements 12 weeks or more after the parents have mastered the method might have revealed child changes related to parent skill in delivering P-ESDM. Child changes reflected on ADOS, MSEL, and Vineland involve much more than increased frequency of one or more proximal, target behaviors. Significant changes on these distal measures involve many behavioral changes, integrated into overall performance, generalized across environments, and performed in a structured situation with a friendly but unfamiliar adult. It may require more time, or more intervention, for low intensity interventions to set in motion the kinds of 
child changes that will be detectable on standardized tests. Additionally, repeated use of these measures 3 months apart may be affected by error, even though test-retest stability in young children with ASD has been examined and found acceptable, and the data from this study also demonstrate excellent stability of symptoms. ${ }^{31}$ Using proximal measures more directly linked to target behaviors, taken within the context of parental interactions, may be more sensitive to short-term treatment-related changes. However, if only direct target behaviors are measured, then the question of more general effects on child development cannot be answered. Optimal designs will combine both types of measures.

A very important challenge to future studies involves recruiting representative groups of families. While almost a third of our sample was non-Caucasian, the majority of our study participant parents were Caucasian (70\%), with at least a college education (60\%), which may limit generalization of our findings. A final design concern that this study raises is our lack of information about the frequency and quality of parent-child interactions occurring regularly at home. We used a naturalistic play interaction in the clinic to examine parentchild interactions, which is common to this type of study. However, measuring parent-child interaction in the general environment in which coaching occurred may have enhanced parent performance through the influence of various setting events. It may well be that the newly learned parent-interaction skills that the P-ESDM parents demonstrated in our assessments did not reflect the quantity or quality of their use of the targeted techniques at home. In order to understand child change over time, we must know what is going on at home. Until then, we will not be able to understand fully the developmental and behavioral effects of parent-delivered interventions for young children with ASD.

These findings need to be integrated with our (2010) report from a randomized controlled trial of ESDM intervention compared to community treatment. ${ }^{20}$ In the 2010 study, children received 24 months of ESDM, more than 15 hours per week in their homes by trained therapists, a total amount of close to 2000 hours, combined with two full years of biweekly parent training. The home therapists used the same teaching procedures as were taught to the parents in P-ESDM, but the home therapists also delivered a specific, individualized curriculum to each child involving 20 or more different learning targets that were updated weekly throughout the two years of treatment. This curriculum content was not taught to the parents in P-ESDM. Therapist fidelity in the 2010 study was monitored in the home, unlike our monitoring of parent fidelity in P-ESDM, which was done in the clinic. The ESDM children in the 2010 study did quite well, with large and significant increases in DQ, adaptive behavior, and language skills, and their gains were similar in size to the gains reported by the UCLA studies of high intensity interventions carried out 1:1 in homes for several years. ${ }^{43}$ Thus, there are many differences between the intervention delivered in the in the efficacious 2010 ESDM study and the P-ESDM study being reported here, which did not yield any group effects compared to the community group. In the present study, both groups of children improved, exhibiting significantly increased DQ scores and decreased autism symptoms in 12 weeks. The role that the interventions played is unknown.

Findings from the present study combined with our 2010 study lead to two important implications and recommendations for provision of early intervention in ASD. First, our findings suggest that the younger the child at the initiation of intervention, even among one year olds, the more developmental gain and symptom reduction occurs over the short term. This provides strong empirical support both for the goal of beginning early intervention as soon as possible, and for the importance of identifying young children at risk for ASD as early as possible. ${ }^{8}$ It is gratifying to see these needs highlighted in the Interagency Autism Coordinating Committee of the National Institutes of Health 2011 Strategic Plan and autism research foundation funding programs. ${ }^{44}$ The resulting recommendation is to move quickly to assess one year olds whose parents or physicians are concerned about ASD. Waiting lists 
for evaluations in tertiary care centers must be replaced by fast-track services so that the youngest children can quickly receive diagnostic assessments and get to ASD-specific services.

The second issue concerns intensity of intervention. It is the practice in many countries and states that relatively few intervention hours are offered to children with ASD profiles before the age of 2 or 3 . However, in our study, those children receiving more hours (within a range from 0 to 15 hours per week) made greater developmental gains in both verbal and nonverbal skills. This suggests that the number of intervention hours matters, even for these young toddlers. Professionals can help families maximize intervention hours by (1) coaching them to provide high quality high frequency learning opportunities at home and (2) steering them to high quality interventions available in the community for their toddlers, as soon as ASD appears. The "wait and see" approach to early ASD must be replaced by an "act now" mentality.

\section{Acknowledgments}

This research was funded by Autism Speaks grants (A.E., S.R.) and by the National Institute of Mental Health (NIMH)/the National Institute of Child Health and Human Development (NICHD) grant MH R01 081757 (S.R.).

We wish to acknowledge the work of the therapists and families involved in this study, and Diane Larzelere of the UC Davis MIND Institute for her help with the manuscript.

\section{References}

1. Warren Z, McPheeters ML, Sathe N, Foss-Feig JH, Glasser A, Veenstra-VanderWeele J. A systematic review of early intensive intervention for autism spectrum disorders. Pediatr. 2011; 127(5):e1303-e1311.

2. Wallace KS, Rogers SJ. Intervening in infancy: Implications for autism spectrum disorders. J Child Psychol Psychiatr. 2010; 51(12):1300-1320.

3. Kasari C, Sigman M, Yirmiya N. Focused and social attention in interactions with familiar and unfamiliar adults: A comparison of autistic, mentally retarded and normal children. Dev Psychopathol. 1993; 5:401-412.

4. Kasari C, Sigman M. Linking parental perceptions to interactions in young children with autism. J Autism Dev Disord. 1997; 27:39-57. [PubMed: 9018581]

5. Moes DR, Frea WD. Contextualized behavioral support in early intervention for children with autism and their families. J Autism Dev Disord. 2002; 32(6):519-533. [PubMed: 12553589]

6. Siller M, Sigman M. The behaviors of parents of children with autism predict the subsequent development of their children's communication. J Autism Dev Disord. 2002; 32(2):77-89. [PubMed: 12058846]

7. Mahoney G, Perales F. Relationship-focused early intervention with children with pervasive developmental disorders and other disabilities: A comparative study. J Dev Behav Pediatr. 2005; 26(2):77-85. [PubMed: 15827458]

8. National Research Council. Educating young children with autism. Washington, DC: National Academy Press; 2001.

9. Koegel RL, Koegel LK, Surratt A. Language intervention and disruptive behavior in preschool children with autism. J Autism Dev Disord. 1992; 22(2):141-153. [PubMed: 1378049]

10. Lutzker, JR.; Huynen, KB.; Bigelow, KM. Parent training. In: Van Hasslet, VB.; Hersen, M., editors. Handbook of psychological treatment protocols for children and adolescents. New Jersey: Lawrence Erlbaum Associates; 1998. p. 467-500.

11. Lutzker, JR.; Steed, SE. Handbook of parent training: Parents as co-therapists for children's behavior problems. 2. Hoboken, NJ: John Wiley and Sons; 1998. Parent training for families of children with developmental disabilities; p. 281-307.

12. Anderson SR, Romanczyk RG. Early intervention for young children with autism: Continuumbased behavioral models. Res Pract Persons Severe Disabl. 1999; 24(3):162-173. 
13. Krantz PJ, MacDuff MT, McClannahan LE. Programming perception in family activities for children with autism: Parents' use of photographic activity schedules. J Appl Behav Anal. 1993; 26(1):137-138. [PubMed: 8473254]

14. Koegel RL, Symon JB, Koegel LK. Parent education for families of children with autism living in geographically distant areas. J Posit Behav Interv. 2002; 4(2):88-103.

15. Laski KE, Charlop MH, Schreibman L. Training parents to use the natural language paradigm to increase their autistic children's speech. J Appl Behav Anal. 1988; 21(4):391-400. [PubMed: 3225256]

16. Stahmer AC. Teaching symbolic play skills to children with autism using pivotal response training. J Autism Dev Disord. 1995; 25(2):123-141. [PubMed: 7559281]

17. Stahmer AC, Schreibman L. Teaching children with autism appropriate play in unsupervised environments using a self-management treatment package. J Appl Beh Anal. 1992; 25(2):447-459.

18. Ingersoll B, Gergans S. The effect of a parent-implemented imitation intervention on spontaneous imitation skills in young children with autism. Res Dev Disabil. 2007; 28(2):163-175. [PubMed: 16603337]

19. Koegel, RL.; Schreibman, L.; Britten, KR.; Burke, JC.; O’Neill, RE. A comparison of parent to direct clinic treatment. In: Koegel, RL.; Rincover, A.; Egel, AL., editors. Educating and understanding autistic children. San Diego: College Hill; 1982. p. 260-279.

20. Dawson G, Rogers S, Munson J, et al. Randomized controlled trial of the Early Start Denver Model: A developmental behavioral intervention for toddlers with autism: Effects on IQ, adaptive behavior, and autism diagnosis. Pediatr. 2010; 125(1):e17-e23.

21. Rogers, SJ.; Dawson, G. The Early Start Denver Model for Young Children with Autism: Promoting language, learning, and engagement. NY: Guilford; 2010.

22. Vismara LA, Colombi C, Rogers SJ. Can 1 hour per week of therapy lead to lasting changes in young children with autism? Autism. 2009; 13(1):93-115. [PubMed: 19176579]

23. Kasari C, Gulsrud AC, Wong C, Kwon S, Locke J. Randomized controlled caregiver mediated joint engagement intervention for toddlers with autism. J Autism Dev Disord. 2010; 40(9):10451056. [PubMed: 20145986]

24. Rogers SJ. Neuropsychology of autism and its implications for early intervention. Mental Retardation and Developmental Disabilities Review. 1998; 4:104-112.

25. Dawson G, Toth K, Abbott R, et al. Early social attention impairments in autism: Social orienting, joint attention and attention to distress. Dev Psychol. 2004; 40(2):271-283. [PubMed: 14979766]

26. Swinkels SHN, Dietz C, van Daalen E, Kerkhof IHGM, van Engeland H, Buitelaar JK. Screening for autistic spectrum in children aged 14to 15 months. I: The development of the early screening of autistic traits questionnaire (ESAT). J Autism Dev Disord. 2006; 36(6):723-732. [PubMed: 16614790]

27. Wetherby, A.; Prizant, B. Communication and symbolic behavior scales developmental profile. Baltimore, MD: Paul H. Brookes; 2002. First Normed edition

28. Robins, D.; Fein, D.; Barton, M. The modified checklist for autism in toddlers (M-CHAT). Retrieved April 2, 2012, from http://www2.gsu.edu/ psydlr/DianaLRobins/Official_MCHAT_Website.html.1999

29. Robins DL. Screening for autism spectrum disorders in primary care settings. Autism. 2008; 12(5): 537-556. [PubMed: 18805946]

30. Robins DL, Fein D, Barton ML, Green JA. The modified checklist for autism in toddlers: An initial study investigating the early detection of autism and pervasive developmental disorders. J Autism Dev Disord. 2001; 31(2):131-144. [PubMed: 11450812]

31. Lord, CE.; Luyster, R.; Gotham, K.; Guthrie, WJ. Autism Diagnostic Observation Schedule Toddler Module. Los Angeles, CA: Western Psychological Services; 2012.

32. Luyster R, Gotham K, Guthrie W, Coffing M, Petrak R, Pierce K, Bishop S, Esler A, Hus V, Oti R, Richler J, Risi S, Lord C. The Autism Diagnostic Observation Schedule-toddler module: a new module of a standardized diagnostic measure for autism spectrum disorders. J Autism Dev Disord. 2009; 39(9):1305-20. [PubMed: 19415479]

33. Mullen, E. The Mullen Scales of Early Learning. Circle Pines, MN: American Guidance; 1995. 
34. Fenson, L.; Marchman, VA.; Thal, D.; Dale, PS.; Bates, E.; Reznick, JS. The macArthur-bates communicative development inventories: Level III: User's guide and technical manual. 2. Baltimore: Brookes; 2007.

35. Yoder P, Stone WL. Randomized comparison of two communication interventions for preschoolers with autism spectrum disorders. J Consult Clin Psychol. 2006; 74(3):426-435. [PubMed: 16822100]

36. Sparrow, S.; Balla, D.; Cichetti, D. Vineland Adaptive Behavior Scales (survey form). Circle Pines, MN: American Guidance Services; 1984.

37. Rogers SJ, Hepburn SL, Stackhouse T, Wehner EA. Imitation performance in toddlers with autism and those with other developmental disorders. J Child Psychol Psychiatr. 2003; 44(5):763-781.

38. Derogatis, L. Symptom checklist-90-revised. San Antonio, Texas: Pearson; 2004.

39. Davis, NO.; Kuhn, JC.; Carter, AS. Children's problem behaviors and self-efficacy among mothers of toddlers with autism. The moderating role of working alliance. Poster presentation, International Meeting of Autism Researchers; Montreal. 2006.

40. Rogers, SJ.; Dawson, G.; Vismara, L. An early start for your child with autism: Using everyday activities to help kids connect, communicate and learn. NY: Guilford; 2012.

41. Hanft, BE.; Rush, DD.; Shelden, ML. Coaching families and colleagues in early childhood. Baltimore, MD: Brookes; 2004.

42. Mahoney G, Perales F. Using relationship-focused intervention to enhance the social-emotional functioning of young children with autism spectrum disorders. Topics Early Child Spec Educ. 2003; 23(2):74-86.

43. McEachin JJ, Smith T, Lovaas OI. Long-term outcome for children with autism who received early intensive behavioral treatment. American Journal of Mental Retardation. 1993; 97:359-372. [PubMed: 8427693]

44. Interagency Autism Coordinating Committee. [Accessed May 1, 2012] The Interagency Autism Coordinating Committee strategic plan for autism spectrum disorder research. Jan 28. 2011 http:// iacc.hhs.gov/strategic-plan/2011/IACC_2011_Strategic_Plan.pdf 
Table 1

Child and family baseline characteristics of 98 toddlers by assignment group.

\begin{tabular}{|c|c|c|}
\hline Variable & $\begin{array}{l}\text { P-ESDM }(n=49) \\
\text { mean }(S D)\end{array}$ & $\begin{array}{l}\text { Community }(n=49) \\
\text { mean }(S D)\end{array}$ \\
\hline Male Gender & $37(75.5)$ & $39(62.5)$ \\
\hline Ethnicity white & $34(69.4)$ & $37(75.5)$ \\
\hline Income $<50 \mathrm{~K}$ & $10(22.2)$ & $15(32.6)$ \\
\hline $50 \mathrm{~K}-75 \mathrm{~K}$ & $5(11.1)$ & $9(19.6)$ \\
\hline $75 \mathrm{~K}-100 \mathrm{~K}$ & $15(33.3)$ & $12(26.1)$ \\
\hline$>100 \mathrm{~K}$ & $15(33.3)$ & $10(21.7)$ \\
\hline Maternal Education Less than high school & $6(12.8)$ & 13(27.1) \\
\hline Some college & $10(21.3)$ & $8(16.7)$ \\
\hline College & $17(36.2)$ & $21(43.8)$ \\
\hline Some graduate school or graduate school & $14(29.8)$ & $6(12.5)$ \\
\hline SCL-90 Primary caretaker ${ }^{*} p<.05$ & $47.05(11.02)$ & $53.47(11.46)$ \\
\hline Child Age/Pre-Treatment (month) & $21.02(3.51)$ & $20.94(3.42)$ \\
\hline Child Age/Post-treatment (month) & $24.77(3.15)$ & $24.49(3.43)$ \\
\hline Time 1 cumulative treatment hours over child's life & $16.16(4.97)$ & $11.06(3.13)$ \\
\hline Time 2 Mean weekly Treatment Hours ${ }^{*} p<.05$ & $1.48(1.94)$ & $3.68(3.91)$ \\
\hline Modified ADOS Social Affect ${ }^{*}$ & $34.14(8.69)$ & $29.45(9.16)$ \\
\hline Imitation Score * & $2.53(2.6)$ & $3.78(3.12)$ \\
\hline \multicolumn{3}{|l|}{ Nonsocial Orient * } \\
\hline 0 & $7(14.3)$ & $8(17.0)$ \\
\hline 0.25 & $6(12.2)$ & $10(21.3)$ \\
\hline 0.5 & $8(16.3)$ & $2(4.3)$ \\
\hline 0.67 & $0(0)$ & $2(4.3)$ \\
\hline 0.75 & $13(26.5)$ & $12(25.5)$ \\
\hline 1 & $15(30.6)$ & $13(27.7)$ \\
\hline
\end{tabular}

Note: ADOS = Autism Diagnostic Observation Schedule; P-ESDM = Parent delivery-Early Start Denver Model; SCL-90 = Symptom Checklist-90.

Two sample Wilcoxon test (continuous variable) or Fisher's exact test (categorical variable) $p$ value $<0.05$ 


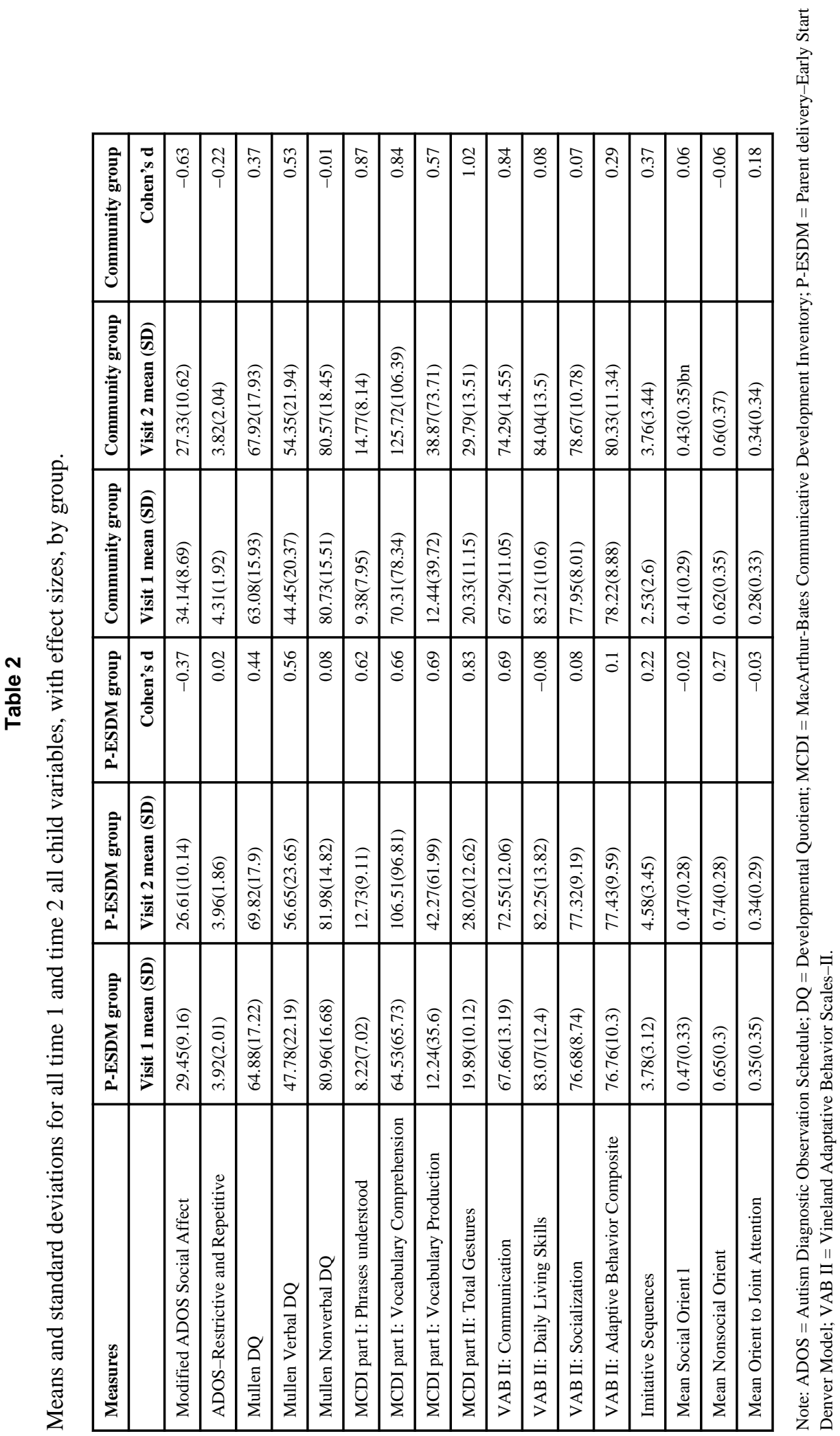


Table 3

Overall and group stratified effect of independent intervention hours on follow-up child outcomes, adjusting for baseline age, modified Autism Diagnostic Observation Schedule social affect and baseline child outcomes, $\mathrm{n}=98$.

\begin{tabular}{|c|c|c|c|c|}
\hline & Overall & P-ESDM Group & Community Group & Interaction $p$ value \\
\hline & Coefficient $(95 \%$ CI $)$ & Coefficient $(95 \%$ CI $)$ & Coefficient $(95 \%$ CI $)$ & \\
\hline Modified ADOS Social Affect & $-0.46(-0.99,0.07)$ & $0.65(-0.46,1.76)$ & $-0.56(-1.22,0.11)$ & 0.166 \\
\hline ADOS Restrictive and Repetitive & $-0.11(-0.22,0) *$ & $-0.1(-0.34,0.14)$ & $-0.08(-0.22,0.06)$ & 0.737 \\
\hline Mullen DQ & $0.78(0.08,1.47) *$ & $1.52(-0.05,3.09)$ & $0.63(-0.25,1.51)$ & 0.289 \\
\hline Mullen Verbal IQ & $1.09(0.11,2.06) *$ & $2.15(-0.04,4.33)$ & $0.81(-0.4,2.03)$ & 0.258 \\
\hline Mullen Nonverbal IQ & $0.43(-0.27,1.13)$ & $0.75(-0.75,2.25)$ & $0.36(-0.57,1.3)$ & 0.644 \\
\hline $\begin{array}{l}\text { MCDI words and Gestures part I: Phrases } \\
\text { understood }\end{array}$ & $0.13(-0.3,0.55)$ & $-0.14(-1.19,0.92)$ & $0.07(-0.42,0.57)$ & 0.702 \\
\hline $\begin{array}{l}\text { MCDI words and Gestures part I: } \\
\text { Vocabulary Comprehension }\end{array}$ & $4.22(0.15,8.3) *$ & $9.99(1.34,18.65) *$ & $1.31(-4.09,6.72)$ & 0.06 \\
\hline $\begin{array}{l}\text { MCDI words and Gestures part I: } \\
\text { Vocabulary Production }\end{array}$ & $1.31(-1.45,4.07)$ & $9.36(3.74,14.97) *$ & $-0.96(-4.47,2.54)$ & 0.005 \\
\hline $\begin{array}{l}\text { MCDI words and Gestures part II: Total } \\
\text { Gestures }\end{array}$ & $0.33(-0.29,0.94)$ & $0.53(-0.92,1.98)$ & $0.14(-0.65,0.93)$ & 0.507 \\
\hline Imitative Sequences Score & $0.17(-0.03,0.37)$ & $0.02(-0.47,0.52)$ & $0.23(-0.01,0.46)$ & 0.469 \\
\hline Social Orient Average Score & $0(-0.02,0.01)$ & $-0.02(-0.06,0.02)$ & $0(-0.03,0.02)$ & 0.616 \\
\hline Nonsocial Orient Average Score & $-0.02(-0.04,0) *$ & $-0.03(-0.07,0.01)$ & $-0.02(-0.03,0)$ & 0.403 \\
\hline Joint Orient: Average Score & $0(-0.02,0.02)$ & $0.01(-0.03,0.05)$ & $-0.01(-0.03,0.02)$ & 0.494 \\
\hline
\end{tabular}

Note: ADOS = Autism Diagnostic Observation Scale for Toddlers; DQ = Developmental Quotient; MCDI = MacArthur-Bates Communicative Development Inventory; P-ESDM = Parent Delivery-Early Start Denver Model.

*

$p$ value $\leq 0.05$ 


\section{Table 4}

Main effect of baseline variables on change scores on Autism Diagnostic Observation Schedule (ADOS) and Mullen Developmental Quotient (DQ), adjusting for baseline age and baseline scores.

\begin{tabular}{|c|c|c|c|}
\hline Change in Outcome & Baseline variable as predictor & Estimated coefficient (SE) & $p$ value \\
\hline \multirow[b]{7}{*}{ Modified ADOS Social Affect } & Modified ADOS Social Affect & $-0.26(0.11)$ & 0.022 \\
\hline & ADOS Restrictive and Repetitive & $0.33(0.44)$ & 0.457 \\
\hline & Mullen DQ & $-0.11(0.07)$ & 0.136 \\
\hline & Imitation Score & $-0.3(0.33)$ & 0.362 \\
\hline & Social Orient Score & $-5.25(2.93)$ & 0.077 \\
\hline & Nonsocial Orient Score & $-7.5(2.8)$ & 0.009 \\
\hline & Joint Attention Score & $-4.92(2.82)$ & 0.085 \\
\hline \multirow[b]{7}{*}{ ADOS Restrictive and Repetitive } & ADOS Restrictive and Repetitive & $0.25(0.1)$ & 0.014 \\
\hline & Modified ADOS Social Affect & $0.02(0.02)$ & 0.371 \\
\hline & Mullen DQ & $-0.01(0.02)$ & 0.444 \\
\hline & Imitation Score & $0(0.07)$ & 0.971 \\
\hline & Social Orient Score & $-1.56(0.65)$ & 0.019 \\
\hline & Nonsocial Orient & $-0.52(0.62)$ & 0.411 \\
\hline & Joint Attention & $-0.39(0.63)$ & 0.536 \\
\hline \multirow[b]{7}{*}{ Mullen DQ } & Mullen DQ & $0.53(0.1)$ & $<0.001$ \\
\hline & Modified ADOS Social Affect & $-0.09(0.16)$ & 0.598 \\
\hline & ADOS Restrictive and Repetitive & $0.04(0.64)$ & 0.947 \\
\hline & Imitation & $0.43(0.48)$ & 0.376 \\
\hline & Social Orient & $1.79(4.24)$ & 0.675 \\
\hline & Nonsocial Orient & $13.04(4.06)$ & 0.002 \\
\hline & Joint Attention & $5.6(4.09)$ & 0.175 \\
\hline
\end{tabular}


Table 5

Regression analysis of assignment group differences in parent-child interaction scores at Time 1 (T1) and Time 2 (T2).

\begin{tabular}{|l|c|l|c|r|}
\hline ESDM & \multicolumn{2}{|l|}{ Control } & t-test for group difference, $\boldsymbol{p}$ value \\
\hline $\mathrm{n}$ & 41 & $\mathrm{n}$ & 40 & 0.127 \\
\hline $\mathrm{T} 1$, mean $(\mathrm{sd})$ & $40.05(5.93)$ & Time 1, mean (sd) & $37.83(6.97)$ & 0.039 \\
\hline $\mathrm{T} 2$, mean $(\mathrm{sd})$ & $45.2(8.68)$ & Time 2, mean (sd) & $41.18(8.53)$ & 0.382 \\
\hline mean change (sd) & $5.14(9.04)$ & mean change (sd) & $3.35(9.35)$ & \\
\hline Cohen' s d & 0.57 & Cohen's d & 0.36 & \\
\hline t-test comparing T1 and T2, $p$ & 0.001 & t-test comparing T1 and T2, $p$ & 0.029 & \\
\hline
\end{tabular}

Note: ESDM = Early Start Denver Model. 